\title{
铁卟啉催化酮和二甲亚砜以及醋酸铵的环化反应：吡啶的一步合成
}

\author{
刘玉峰 ${ }^{a}$ 曹重仲 ${ }^{a}$ 苏沝东 ${ }^{a}$ 李 慧 $a$ \\ 符美强 ${ }^{a}$ 刘 强 ${ }^{a}$ 罗伟平 $a$ 郭灿城*, \\ ( ${ }^{a}$ 湖南大学化学化工学院 教育部先进催化工程研究中心 长沙 410082)
}

\begin{abstract}
摘要 发展了一种在铁卟啉催化下酮和二甲亚砜(DMSO)以及醋酸铵的环化反应, 该反应利用 DMSO 作为碳源来合成 不对称和对称的吡啶化合物, 多种酮能够很好地和 DMSO 反应产生吡啶化合物，产率在 30\%到 $85 \%$ 之间. 该方法利用 非贵金属和简单酮类化合物来合成吡啶，条件温和，操作简单. 在初步的控制实验基础上，提出了该反应可能的机理. 关键词 吡啶; 铁卟啉; 二甲亚砜; 酮
\end{abstract}

\section{Iron(III) Porphyrin Catalyzed Cyclization of Ketones with Dimethyl Sulfoxide and Ammonium Acetate: One-Pot Synthesis of Pyridines}

\author{
Liu, Yufeng ${ }^{a}$ \\ Cao, Zhongzhong ${ }^{a}$ \\ $\mathrm{Su}$, Miaodong ${ }^{a}$ \\ $\mathrm{Li}, \mathrm{Hui}^{a}$ \\ $\mathrm{Fu}$, Meiqiang ${ }^{a}$ \\ Liu, Qiang ${ }^{a}$ \\ Luo, Weiping ${ }^{a}$ \\ Guo, Cancheng*,a \\ ( ${ }^{a}$ Advanced Catalytic Engineering Research Center of the Ministry of Education, College of Chemistry and \\ Chemical Engineering, Hunan University, Changsha 410082)
}

\begin{abstract}
An iron(III)-porphyrin-catalyzed cyclization of ketones with dimethyl sulfoxide (DMSO) and ammonium acetate for the synthesis of unsymmetrical and symmetrical pyridines by employing DMSO as $\mathrm{C}_{4}$ or $\mathrm{C}_{6}$ source has been developed. Various aryl ketone derivatives react readily with DMSO, producing the pyridines in yields of $30 \% \sim 85 \%$. This method uses non-noble metals and proceeds under mild reaction conditions with operational simplicity, which thus allows the expedient assembly of pyridines from readily available ketones. Based on the preliminary experiments, a plausible mechanism of this transformation is disclosed.
\end{abstract}

Keywords pyridine; iron(III) porphyrin; dimethyl sulfoxide; ketone

\section{Introduction}

Pyridines are important fundamental heterocycles which are found in numerous natural products, functional materials, agrochemicals and pharmaceutical drugs. ${ }^{[1]}$ Molecules bearing a pyridine moiety exhibit a wide range of biological activities, such as anxiolytic, ${ }^{[2]}$ antidiabetic, ${ }^{[3]}$ antiviral, ${ }^{[4]}$ antibacterial,${ }^{[5]}$ antileishmanial,${ }^{[6]}$ anti-inflammatory, ${ }^{[7]}$ and anti-chagasic activities. ${ }^{[8]}$ In addition, pyridine dervatives are extensively used as various valuable ligands (such as bipyridine and terpyridine), organic bases and catalysts. ${ }^{[9]}$ As a result, the synthesis of pyridine and its derivatives has attracted the attention of members of the scientific community for more than 140 years. ${ }^{[10]}$

In recent years, metalloporphyrins have been used as cytochrome P-450 models and have been found to be highly efficient homogeneous or heterogeneous catalysts for the oxidation reactions of organic compounds. ${ }^{[11]}$ Metalloporphyrin-mediated hydrocarbon functionalization is of particular importance in biomimetic investigation and is potentially useful in organic synthesis. The epoxidation, aziridination, and cyclopropanation of alkenes and the hydroxylation and amidation of saturated $\mathrm{C}-\mathrm{H}$ bonds are among the most common hydrocarbon-functionalization reactions mediated by a metalloporphyrin, which are widely believed to proceed by oxygen-, nitrogen-, and carbon-atom ${ }^{[12]}$ transfer from oxo-, imido-, and carbenemetalloporphyrin active species, respectively. ${ }^{[13]}$ In this field, our group has reported some work about metalloporphyrins catalyzed hydrocarbons convert into value-

\footnotetext{
* Corresponding author. E-mail: ccguo@hnu.edu.cn

Received September 7, 2018; revised November 29, 2018; published online December 5, 2018.

Dedicated to Professor Qingyun Chen on the Occasion of his 90th birthday.

Project supported by the National Natural Science Foundations of China (No. 21572049).

国家自然科学基金(No. 21572049)资助项目.
} 
added functional molecules through $\mathrm{C}-\mathrm{O}, \mathrm{C}-\mathrm{N}$ and $\mathrm{C}-$ $\mathrm{C}$ bond formation ${ }^{[14]}$. As a part of our continuous efforts for metalloporphyrin-based atom/group transfer catalysis, herein, we report the first examples of the iron(III)-porphyrin-catalyzed cyclization of ketones with dimethyl sulfoxide (DMSO) and ammonium acetate for the synthesis of unsymmetrical and symmetrical pyridines by employing DMSO as $\mathrm{C}_{4}$ or $\mathrm{C}_{6}$ source, respectively. ${ }^{[15]}$ This method uses non-noble metals and proceeds under mild reaction conditions with operational simplicity, which thus allows the expedient and atom-economical assembly of pyridines from readily available ketones.

\section{Results and discussion}

We first surveyed the catalytic cyclization of ketones by various metalloporphyrins with different nitrogen sources. To our delight, acetophenone (1a) could be converted into pyridine (2a) in $72 \%$ yield with 3.0 equiv. of $\mathrm{NH}_{4} \mathrm{OAc}$ and $1.0 \mathrm{~mol} \% \mathrm{~T}(\mathrm{p}-\mathrm{OMe}) \mathrm{PPFeCl}$ in DMSO under $101 \mathrm{kPa} \mathrm{O}_{2}$ at $120{ }^{\circ} \mathrm{C}$. The results in Table 1 showed that other iron(III)-porphyrin catalysts, including $\mathrm{T}(\mathrm{p}-\mathrm{Cl}) \mathrm{PPFeCl}$, $\mathrm{T}(\mathrm{p}-\mathrm{Me}) \mathrm{PPFeCl}$ and $\mathrm{TPPFeCl}$ could also afford the product, but with less efficiency (Table 1, Entries 1 4). On the other hand, other metal complexes of T( $p-\mathrm{MeO}) \mathrm{PP}$ such as $\mathrm{T}(p-\mathrm{MeO}) \mathrm{PPCu}, \mathrm{T}(p-\mathrm{MOe}) \mathrm{PPC}, \mathrm{T}(p-\mathrm{MeO}) \mathrm{PPMnCl}$ and $\mathrm{T}(p-\mathrm{MeO}) \mathrm{PPNi}$ were also tested, and the results demon-

Table 1 Optimization of reaction conditions

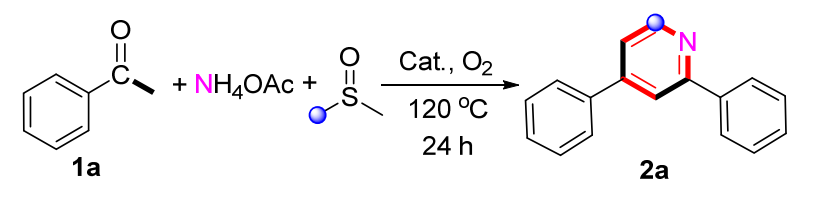

\begin{tabular}{cllcc}
\hline Entry & \multicolumn{1}{c}{$\mathrm{N}$ source $^{\text {Catalyst }}$} & Temp. $/^{\circ} \mathrm{C}$ & Yield $^{b} / \%$ \\
\hline 1 & $\mathrm{NH}_{4} \mathrm{OAc}$ & $\mathrm{T}(p-\mathrm{MeO}) \mathrm{PPFeCl}$ & 120 & 72 \\
2 & $\mathrm{NH}_{4} \mathrm{OAc}$ & $\mathrm{T}(p-\mathrm{Cl}) \mathrm{PPFeCl}$ & 120 & 65 \\
3 & $\mathrm{NH}_{4} \mathrm{OAc}$ & $\mathrm{T}(p-\mathrm{Me}) \mathrm{PPFeCl}$ & 120 & 67 \\
4 & $\mathrm{NH}_{4} \mathrm{OAc}$ & $\mathrm{TPPFeCl}$ & 120 & 62 \\
5 & $\mathrm{NH}_{4} \mathrm{OAc}$ & - & 120 & 12 \\
6 & $\mathrm{NH}_{4} \mathrm{OAc}$ & $\mathrm{T}(p-\mathrm{MeO}) \mathrm{PPCu}$ & 120 & 51 \\
7 & $\mathrm{NH}_{4} \mathrm{OAc}$ & $\mathrm{T}(p-\mathrm{MeO}) \mathrm{PPCo}$ & 120 & 38 \\
8 & $\mathrm{NH}_{4} \mathrm{OAc}$ & $\mathrm{T}(p-\mathrm{MeO}) \mathrm{PPMnCl}$ & 120 & 42 \\
9 & $\mathrm{NH}_{4} \mathrm{OAc}$ & $\mathrm{T}(p-\mathrm{MeO}) \mathrm{PPNi}$ & 120 & 26 \\
10 & $\left.\mathrm{NH}_{4}\right)_{2} \mathrm{CO}$ & $\mathrm{T}(p-\mathrm{MeO}) \mathrm{PPFeCl}$ & 120 & 42 \\
11 & $\mathrm{NH}_{4} \mathrm{HCO}$ & $\mathrm{T}(p-\mathrm{MeO}) \mathrm{PPFeCl}$ & 120 & 40 \\
12 & $\mathrm{NH}_{3} \cdot \mathrm{H}_{2} \mathrm{O}$ & $\mathrm{T}(p-\mathrm{MeO}) \mathrm{PPFeCl}$ & 120 & 36 \\
13 & $\mathrm{NH}_{4} \mathrm{I}$ & $\mathrm{T}(p-\mathrm{MeO}) \mathrm{PPFeCl}$ & 120 & 18 \\
14 & $\mathrm{HCOONH}_{4}$ & $\mathrm{~T}(p-\mathrm{MeO}) \mathrm{PPFeCl}$ & 120 & 65 \\
15 & $\mathrm{NH}_{4} \mathrm{OAc}$ & $\mathrm{T}(p-\mathrm{MeO}) \mathrm{PPFeCl}$ & 100 & 47 \\
16 & $\mathrm{NH}_{4} \mathrm{OAc}$ & $\mathrm{T}(p-\mathrm{MeO}) \mathrm{PPFeCl}$ & 130 & 70 \\
$17^{c}$ & $\mathrm{NH}_{4} \mathrm{OAc}$ & $\mathrm{T}(p-\mathrm{MeO}) \mathrm{PPFeCl}$ & 120 & 63 \\
$18^{d}$ & $\mathrm{NH}_{4} \mathrm{OAc}$ & $\mathrm{T}(p-\mathrm{MeO}) \mathrm{PPFeCl}$ & 120 & 69 \\
\hline
\end{tabular}

${ }^{a}$ Reaction conditions: acetophenone $(1 \mathrm{a}, 0.5 \mathrm{mmol}), \mathrm{N}$ source $(1.5 \mathrm{mmol})$, catalyst $(1.0 \mathrm{~mol} \%)$ and solvent $(2 \mathrm{~mL})$ for $24 \mathrm{~h}$ under $\mathrm{O}_{2}$ atmosphere at $120{ }^{\circ} \mathrm{C} .{ }^{b}$ Isolated yield. ${ }^{c} \mathrm{~T}(p-\mathrm{MeO}) \mathrm{PPFeCl}(0.5 \mathrm{~mol} \%) .{ }^{d} \mathrm{~T}(p-\mathrm{MeO}) \mathrm{PPFeCl}$ $(2.0 \mathrm{~mol} \%)$ strated that $\mathrm{T}(p-\mathrm{MeO}) \mathrm{PPFeCl}$ was the best choice (Table 1, Entries 5 8). When other nitrogen sources were used instead of $\mathrm{NH}_{4} \mathrm{OAc}$, none showed higher efficiency than $\mathrm{NH}_{4} \mathrm{OAc}$ (Table 1, Entries 9 13). Decreased yield was observed when a lower or higher catalyst loading was applied (Table 1, Entries 14, 15).

Subsequently, the scope of the (hetero)aryl methyl ketones was investigated under the standard reaction conditions (Table 2). The results revealed that aryl methyl ketones bearing a variety of functional groups and substitution patterns afforded the desired products in moderate to good yields $(34 \% \sim 82 \%)$. As shown in Table 1 , acetophenone derivatives bearing electrondonating substituents $(\mathrm{MeO}, \mathrm{EtO}, \mathrm{Me})$ could achieve this conversion in $42 \%$ $82 \%$ yields (Table 2 , Entries $1 \sim 8$ ). Nevertheless, the acetophenone derivatives with electron-withdrawing substituents $\left(\mathrm{F}, \mathrm{Cl}, \mathrm{Br}, \mathrm{I}, \mathrm{NO}_{2}, \mathrm{CN}, \mathrm{CF}_{3}\right)$ could be converted into pyridines in yields ranging from $34 \%$ to $70 \%$ (Table 2 , Entries 9 17). Furthermore, acetophenone bearing the same substituents at different positions had influence on the efficiency of this transformation. For example, 2methylacetophenone gave lower yield than 4-/3methylacetophenone (Table 2, Entries $4 \sim 6$ ). Importantly, halogen substituents on the phenyl ring were well tolerated

Table 2 Scope of acetophenones

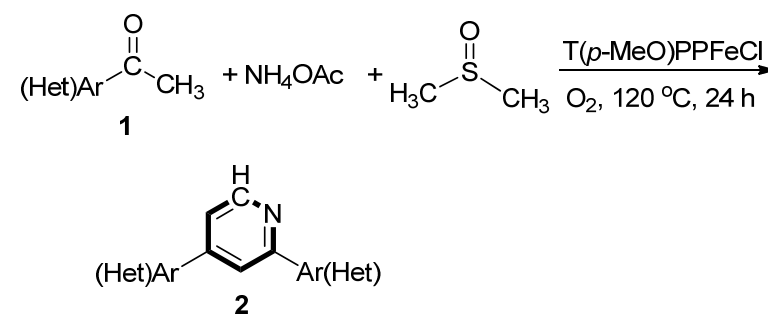

\begin{tabular}{|c|c|c|c|}
\hline Entry & (Het)Ar & 2 & Yield/\% \\
\hline 1 & $\mathrm{Ph}$ & $2 \mathbf{a}$ & 72 \\
\hline 2 & 4- $\mathrm{MeOC}_{6} \mathrm{H}_{4}$ & $2 b$ & 81 \\
\hline 3 & 4-EtOC ${ }_{6} \mathrm{H}_{4}$ & $2 \mathrm{c}$ & 85 \\
\hline 4 & $4-\mathrm{MeC}_{6} \mathrm{H}_{4}$ & 2d & 78 \\
\hline 5 & $3-\mathrm{MeC}_{6} \mathrm{H}_{4}$ & $2 e$ & 73 \\
\hline 6 & $2-\mathrm{MeC}_{6} \mathrm{H}_{4}$ & $2 f$ & 42 \\
\hline 7 & $3,4-(\mathrm{MeO}){ }_{2} \mathrm{C}_{6} \mathrm{H}_{3}$ & $2 \mathrm{~g}$ & 82 \\
\hline 8 & $2,4-(\mathrm{MeO}){ }_{2} \mathrm{C}_{6} \mathrm{H}_{3}$ & $2 \mathrm{~h}$ & 71 \\
\hline 9 & $4-\mathrm{FC}_{6} \mathrm{H}_{4}$ & $2 \mathrm{i}$ & 58 \\
\hline 10 & $4-\mathrm{ClC}_{6} \mathrm{H}_{4}$ & $2 \mathbf{j}$ & 70 \\
\hline 11 & 4- $\mathrm{BrC}_{6} \mathrm{H}_{4}$ & $2 k$ & 66 \\
\hline 12 & $3-\mathrm{BrC}_{6} \mathrm{H}_{4}$ & 21 & 54 \\
\hline 13 & 2- $\mathrm{BrC}_{6} \mathrm{H}_{4}$ & $2 m$ & 34 \\
\hline 14 & $4-\mathrm{IC}_{6} \mathrm{H}_{4}$ & $2 n$ & 54 \\
\hline 15 & $3-\mathrm{NO}_{2} \mathrm{C}_{6} \mathrm{H}_{4}$ & 20 & 35 \\
\hline 16 & $4-\mathrm{CNC}_{6} \mathrm{H}_{4}$ & $2 p$ & 66 \\
\hline 17 & 4- $\mathrm{CF}_{3} \mathrm{C}_{6} \mathrm{H}_{4}$ & $2 q$ & 58 \\
\hline 18 & 2-Naphthyl & $2 r$ & 68 \\
\hline 19 & Furyl & $2 s$ & 52 \\
\hline
\end{tabular}

$\operatorname{PPFeCl}(1.0 \mathrm{~mol} \%)$, DMSO $(2.0 \mathrm{~mL}), 120{ }^{\circ} \mathrm{C}$ for $24 \mathrm{~h} .{ }^{b}$ Isolated yield. 
with this conversion, which enable a potential application in further functionalization (Table 2, Entries 9 14). Furthermore, 2-acetonaphthone and 2-acetylthiophene could also provide the desired product in moderate yield (Table 2, Eentries 18 and 19).

It is worth noting that the substrates of $\mathbf{1 f}, \mathbf{1 m}$ and $\mathbf{1 0}$ could also gave the corresponding products, but the yields are only $42 \%, 34 \%$ and $35 \%$, respectively. With further research, we found that the symmetrical pyridines were obtained and the results are in contrary to the previous literature which only unsymmetrical pyridines were obtained (Table 3). ${ }^{[15 \mathrm{~b}]}$

Table 3 Scope of acetophenones

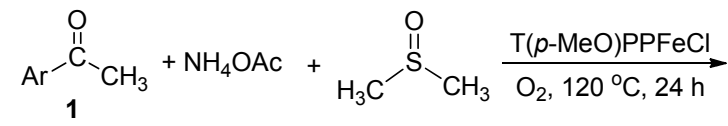

$$
\begin{aligned}
& \overbrace{2}^{C} C_{A r}^{C}+C_{A r}^{C} \\
& \text { 2' }
\end{aligned}
$$

\begin{tabular}{clccl}
\hline \multirow{2}{*}{ Entry } & \multirow{2}{*}{$\mathrm{Ar}$} & $\mathbf{1}$ & \multicolumn{2}{c}{ Yield/\% } \\
\cline { 4 - 5 } & & & \multicolumn{1}{c}{$\mathbf{2}^{a}$} & \multicolumn{1}{c}{$\mathbf{2}^{b}$} \\
\hline 1 & $2-\mathrm{CH}_{3} \mathrm{C}_{6} \mathrm{H}_{4}$ & $\mathbf{1 f}$ & $42(\mathbf{2 f})$ & $40\left(\mathbf{2 f ^ { \prime }}\right)$ \\
2 & $2-\mathrm{BrC}_{6} \mathrm{H}_{4}$ & $\mathbf{1 m}$ & $34(\mathbf{2 m})$ & $38\left(\mathbf{2} \mathbf{m}^{\prime}\right)$ \\
3 & $3-\mathrm{NO}_{2} \mathrm{C}_{6} \mathrm{H}_{4}$ & $\mathbf{1 0}$ & $35(\mathbf{2 o})$ & $30(\mathbf{2 o})$ \\
\hline
\end{tabular}

${ }^{a}$ Standard conditions: ketones $(0.5 \mathrm{mmol}), \mathrm{NH}_{4} \mathrm{OAc}$ (3.0 equiv.), $\mathrm{T}(p-\mathrm{MeO})-$ PPFeCl (1.0 mol\%), DMSO (2.0 mL), $120{ }^{\circ} \mathrm{C}$ for $24 \mathrm{~h} .{ }^{b}$ Isolated yield.

To expand the substrate scope of this transformation, $\alpha$-substituent on the ketones was investigated and the results are displayed in Table 4. Surprisingly, the substitution patterns of the pyridine ring changed when propiophenone was used as a substrate instead of an aryl methyl ketone. Based on this result, we proceeded to investigate a variety of other propiophenone compounds. As expected, the electronic natue of the phenyl ring on the propiophenone compound had little impact on the efficiency of the reaction $(54 \% \sim 71 \%$ yields for $\mathbf{1 t} \sim \mathbf{1 w})$. Moreover, the use of valerophenone, 1,3-diphenylpropane-1,3-dione or benzocyclohexanone as a substrate under the optimized conditions also gave the corresponding products $\mathbf{3 e}, \mathbf{3 f}$ and $\mathbf{3 g}$ in moderate to good yields $(54 \% \sim 65 \%)$.

In order to have a deeper understanding on the mechanism, several control experiments were conducted (Scheme 1). The reaction of hydrated hemiacetal (1ab) or 1-phenyl2-propen-1-one (1ac) with ammonium acetate under the standard conditions, did not lead to the desired product $\mathbf{2 a}$ (Scheme 1), which suggested that these two substrates were not intermediates in the reaction. To lend further support to this mechanism, we investigated the preparation and subsequent reaction of substrates 1 ad and 1ae. The results revealed that 1 ad and 1 ae could be converted to the desired products, albeit in a lower yield (Scheme 1), which clearly confirmed that $\mathbf{1 a d}$ and 1ae were not the key intermediates in the transformation. The use of DMSO- $d_{6}$
Table 4 Scope of $\alpha$-substituent ketones

\begin{tabular}{|c|c|c|c|c|c|}
\hline Entry & $\mathrm{R}^{1}$ & $\mathrm{R}^{2}$ & 1 & 3 & Yield $^{b} / \%$ \\
\hline 1 & $\mathrm{Ph}$ & $\mathrm{Me}$ & 1t & $3 \mathbf{a}$ & 65 \\
\hline 2 & $4-\mathrm{MeOC}_{6} \mathrm{H}_{4}$ & $\mathrm{Me}$ & $1 \mathrm{u}$ & $3 \mathbf{b}$ & 71 \\
\hline 3 & $4-\mathrm{MeC}_{6} \mathrm{H}_{4}$ & $\mathrm{Me}$ & $1 v$ & $3 c$ & 68 \\
\hline 4 & $4-\mathrm{ClC}_{6} \mathrm{H}_{4}$ & $\mathrm{Me}$ & $1 w$ & 3d & 60 \\
\hline 5 & $\mathrm{Ph}$ & $n-\operatorname{Pr}$ & $1 x$ & $3 e$ & 54 \\
\hline 6 & $\mathrm{Ph}$ & $\mathrm{PhC}=\mathrm{O}$ & $1 y$ & $3 f$ & 65 \\
\hline 7 & $\mathrm{CH}_{2}=\mathrm{CHC}_{6} \mathrm{H}_{4}$ & $\mathrm{H}$ & $1 z$ & & 0 \\
\hline 8 & $\mathrm{Ph}$ & $\mathrm{Ph}$ & $1 \mathrm{af}$ & & 0 \\
\hline 9 & & & 1 ag & $3 g$ & 64 \\
\hline 10 & & & 1ah & & 0 \\
\hline
\end{tabular}

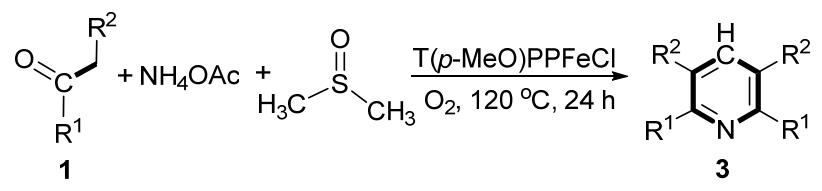

${ }^{a}$ Standard conditions: ketones $(0.5 \mathrm{mmol}), \mathrm{NH}_{4} \mathrm{OAc}$ (3.0 equiv.), $\mathrm{T}(p$-OMe)PPFeCl (1.0 mol\%), DMSO $(2.0 \mathrm{~mL}), 120{ }^{\circ} \mathrm{C}$ for $24 \mathrm{~h} .{ }^{b}$ Isolated yield.

in the reaction showed that DMSO behaved as a source of methylene units, following the isolation of the partially deuterated products 2a- $d_{1}$ and 3a- $d_{1}$ (Scheme 1), which further confirmed that DMSO was a source of methylene units.

On the basis of the experimental results described above and previous literatures, ${ }^{[15,16]}$ we propose a possible mechanism for this transformation (Figure 1). The initial reaction of carbonyl group of ketones (1a) with ammonia from ammonium acetate would give intermediate A. Contrary to the previous literature of two mechanisms to obtain unsymmetrical or symmetrical product, ${ }^{[23]}$ the intermediate $\mathbf{A}$ would undergo two pathway to give unsymmetrical or symmetrical pyridines respectively. DMSO could be protonated by acetic acid which was formed in-situ from ammonium acetate to give an activated species, which would be converted to the methylene source B. When the substrates were methyl ketones, the subsequent nucleophilic attack of $\mathbf{B}$ by the nitrogen atom of $\mathbf{A}$ would lead to the formation of intermediate $\mathbf{C}$. Intermediate $\mathbf{C}$ would then undergo a rapid nucleophilic attack on acetophenone and through a dehydration reaction to afford $\mathbf{D}$. The elimination of methanethiol, followed by sequential $\mathbf{6 p}$ electrocyclization and aromatization ${ }^{[14]}$ reactions to provide the desired product $\mathbf{2 a}$. On the other hand, when the substrates were $\alpha$-substituent ketones, the subsequent nucleophilic attack of $\mathbf{B}$ by the $\alpha$-carbon atom of $\mathbf{A}$ would lead to the formation of intermediate $\mathbf{E}$. Intermediate $\mathbf{E}$ would then undergo a rapid nucleophilic attack on $\alpha$-substituent ketones and through a dehydration reaction to afford F. Finally, annulation and aromatization of $\mathbf{F}$ would then give 

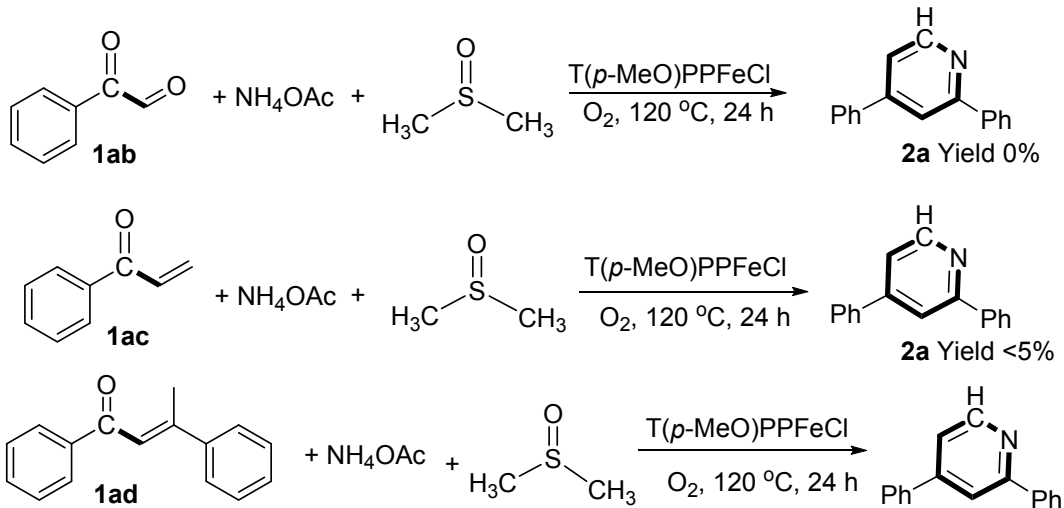

2a Yield $<5 \%$
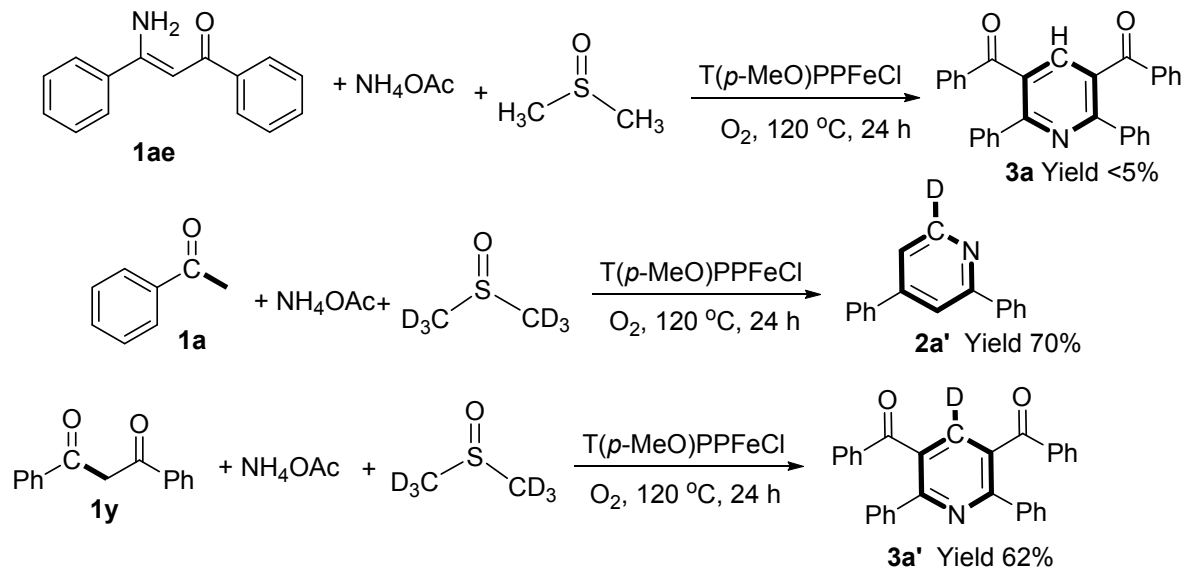

Scheme 1 Control experiments

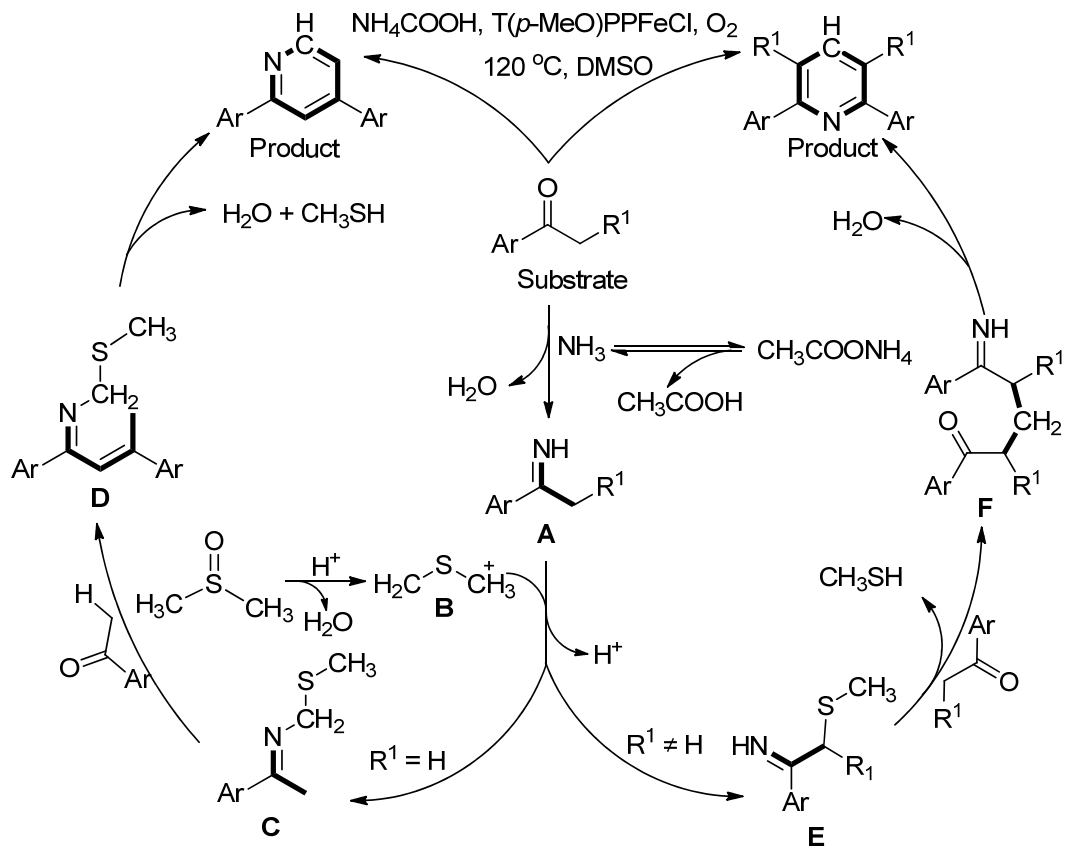

Figure 1 Possible mechanism

the desired product $\mathbf{3 a}$.

\section{Conclusions}

In summary, we have developed a novel and efficient methodology for the synthesis of diverse and functionalized pyridines in good yield by iron(III)-porphyrincatalyzed three-component reactions of readily available ketones with DMSO and ammonium acetate. The method- 
ology provides an environmentally friendly, simple and regioselective access to a variety of multisubstituted pyridines. This protocol tolerates a wide range of substituents, heteroatoms, and steric environments to provide polysubstituted pyridine derivatives and forms various $\mathrm{C}-\mathrm{C}$ and $\mathrm{C}-\mathrm{N}$ bonds in a single procedure.

\section{Experimental section}

\subsection{General methods}

Materials obtained from commercial suppliers were used as received unless mentioned otherwise. Products were purified by flash chromatography on silica gel $(300 \sim 400$ mesh), and were characterized by ${ }^{1} \mathrm{H} N M R$, and ${ }^{13} \mathrm{C} \mathrm{NMR}$. ${ }^{1} \mathrm{H}$ NMR spectra were recorded on $400 \mathrm{MHz}$ NMR spectrometer. ${ }^{13} \mathrm{C}$ NMR spectra were obtained at $100 \mathrm{MHz}$ and referenced to the internal solvent signals (central peak is $\delta$ 77.0 in $\mathrm{CDCl}_{3}$ ). High-resolution mass spectra (HRMS) were measured on an electrospray ionization (ESI) apparatus using time-of-flight ( $\mathrm{ToF}$ ) mass spectrometry.

\subsection{Typical experimental procedure for the formation} of pyridines from arylketones

In a Schlenk tube of $25 \mathrm{~mL}, \mathrm{CH}_{3} \mathrm{COONH}_{4}(1.5 \mathrm{mmol}$, 3.0 equiv.), acetophenone 1 ( $0.5 \mathrm{mmol}, 1.0$ equiv.) and $\mathrm{T}(p$-OMe)PPFeCl $(1.0 \mathrm{mmol} \%)$ were dissolved in DMSO $(2 \mathrm{~mL})$ and stirred at $120{ }^{\circ} \mathrm{C}$ for $24 \mathrm{~h}$. After completion of the reaction. The resulting solution was cooled to room temperature. The solution was diluted with ethyl acetate $(10 \mathrm{~mL})$, washed with water $(5 \mathrm{~mL})$, extracted with ethyl acetate $(5 \mathrm{~mL} \times 3)$, and dried over anhydrous $\mathrm{Na}_{2} \mathrm{SO}_{4}$ and concentrated in vacuo. The crude product was purified by flash column chromatography on silica gel to give the desired product.

2,4-Diphenylpyridine (2a): Yellow solid, $42 \mathrm{mg}, 72 \%$ yield. m.p. $54 \sim 58{ }^{\circ} \mathrm{C}$; ${ }^{1} \mathrm{H}$ NMR $\left(\mathrm{CDCl}_{3}, 400 \mathrm{MHz}\right) \delta$ : $8.74(\mathrm{~d}, J=5.1 \mathrm{~Hz}, 1 \mathrm{H}), 8.05(\mathrm{~d}, J=7.7 \mathrm{~Hz}, 2 \mathrm{H}), 7.92$ (s, $1 \mathrm{H}), 7.69$ (d, $J=7.6 \mathrm{~Hz}, 2 \mathrm{H}), 7.55 \sim 7.40(\mathrm{~m}, 7 \mathrm{H}) ;{ }^{13} \mathrm{C}$ NMR $\left(\mathrm{CDCl}_{3}, 100 \mathrm{MHz}\right) \delta: 158.1,150.1,150.0,149.4$, $139.5,138.6,129.2,129.1,128.8,127.1,127.0,120.3$, 118.8; IR (KBr) v: 1605, 1593, 1578, 1540, 1467, 1387, $774,728,694,610 \mathrm{~cm}^{-1}$; HRMS (EI) calcd for $\mathrm{C}_{17} \mathrm{H}_{14} \mathrm{~N}$ : 232.1125 , found 232.1123 .

2,4-Bis(4-methoxyphenyl)pyridine (2b): White solid, 59 mg, 81\% yield. m.p. $131 \sim 134{ }^{\circ} \mathrm{C} ;{ }^{1} \mathrm{H}$ NMR $\left(\mathrm{CDCl}_{3}, 400\right.$ MHz) $\delta: 8.69(\mathrm{~d}, J=5.0 \mathrm{~Hz}, 1 \mathrm{H}), 7.94(\mathrm{~d}, J=7.6 \mathrm{~Hz}, 2 \mathrm{H})$, 7.89 (s, 1H), 7.59 (d, $J=7.6 \mathrm{~Hz}, 2 \mathrm{H}), 7.40$ (d, $J=5.0 \mathrm{~Hz}$, 1H), $7.30(\mathrm{~d}, J=7.7 \mathrm{~Hz}, 4 \mathrm{H}), 2.42(\mathrm{~s}, 6 \mathrm{H}) ;{ }^{13} \mathrm{C} \mathrm{NMR}$ $\left(\mathrm{CDCl}_{3}, 100 \mathrm{MHz}\right) \delta: 158.0,149.9,149.7,149.2,139.2$, $139.1,136.7,135.7,129.9,129.5,126.9,119.8,118.3$, 21.3, 21.2; IR (KBr) v: 1607, 1595, 1514, 1469, 1251, 1238, 1183, 1043, 1019, 827, $815 \mathrm{~cm}^{-1}$; HRMS (EI) calcd for $\mathrm{C}_{19} \mathrm{H}_{18} \mathrm{NO}_{2} 292.1332$, found 292.1331.

2,4-Bis(4-ethoxyphenyl)pyridine (2c): White solid, 68 mg, 85\% yield. m.p. $135 \sim 138{ }^{\circ} \mathrm{C}$; ${ }^{1} \mathrm{H}$ NMR $\left(\mathrm{CDCl}_{3}, 400\right.$ MHz) $\delta: 8.58(\mathrm{~d}, J=4.9 \mathrm{~Hz}, 1 \mathrm{H}), 7.90(\mathrm{~d}, J=8.3 \mathrm{~Hz}, 2 \mathrm{H})$, 7.75 (s, 1H), 7.55 (d, $J=8.3 \mathrm{~Hz}, 2 \mathrm{H}), 7.28(\mathrm{~d}, J=4.9 \mathrm{~Hz}$, 1H), 6.92 (d, $J=8.3 \mathrm{~Hz}, 4 \mathrm{H}), 4.02$ (q, $J=6.8 \mathrm{~Hz}, 4 \mathrm{H}), 1.37$ $(\mathrm{t}, J=6.4 \mathrm{~Hz}, 6 \mathrm{H}) ;{ }^{13} \mathrm{C} \mathrm{NMR}\left(\mathrm{CDCl}_{3}, 100 \mathrm{MHz}\right) \delta: 159.9$, $157.6,149.7,148.9,131.9,130.6,128.4,128.2,119.1$, $117.6,115.1,114.7,114.0,63.7,63.6,14.9,14.8 ; \mathrm{IR}(\mathrm{KBr})$ $v: 2978,1601,1541,1513,1465,1396,1254,1239,1181$, 1116, 1048, 828, $809 \mathrm{~cm}^{-1}$; HRMS (EI) calcd for $\mathrm{C}_{21} \mathrm{H}_{22} \mathrm{NO}_{2}$ 320.1645, found 320.1647.

2,4-Di-p-tolylpyridine (2d): White solid, $51 \mathrm{mg}, 78 \%$ yield. m.p. $104 \sim 106{ }^{\circ} \mathrm{C} ;{ }^{1} \mathrm{H}$ NMR $\left(\mathrm{CDCl}_{3}, 400 \mathrm{MHz}\right) \delta$ : 8.69 (d, $J=5.0 \mathrm{~Hz}, 1 \mathrm{H}), 7.94$ (d, $J=7.6 \mathrm{~Hz}, 2 \mathrm{H}), 7.89$ (s, 1H), 7.59 (d, $J=7.6 \mathrm{~Hz}, 2 \mathrm{H}), 7.40$ (d, $J=5.0 \mathrm{~Hz}, 1 \mathrm{H}), 7.30$ $(\mathrm{d}, J=7.7 \mathrm{~Hz}, 4 \mathrm{H}), 2.42(\mathrm{~s}, 6 \mathrm{H}) ;{ }^{13} \mathrm{C} \mathrm{NMR}\left(\mathrm{CDCl}_{3}, 100\right.$ MHz) $\delta: 158.0,149.9,149.2,139.2,139.1,136.7,135.7$, $129.9,129.5,126.9,119.8,118.3,21.3,21.2$; IR (KBr) $v$ : 1596, 1539, 1515, 1470, 1382, 808, 747, 527, $436 \mathrm{~cm}^{-1}$; HRMS (EI) calcd for $\mathrm{C}_{19} \mathrm{H}_{18} \mathrm{~N} 260.1434$, found 260.1436 .

2,4-Di- $m$-tolylpyridine (2e): Yellow oil, $47 \mathrm{mg}, 73 \%$ yield. ${ }^{1} \mathrm{H} \mathrm{NMR}\left(\mathrm{CDCl}_{3}, 400 \mathrm{MHz}\right) \delta: 8.71(\mathrm{~d}, J=5.1 \mathrm{~Hz}$, 1H), 7.90 (d, $J=6.9 \mathrm{~Hz}, 2 \mathrm{H}), 7.82(\mathrm{~d}, J=7.7 \mathrm{~Hz}, 1 \mathrm{H}), 7.49$ $(\mathrm{d}, J=7.0 \mathrm{~Hz}, 2 \mathrm{H}), 7.43 \sim 7.36(\mathrm{~m}, 3 \mathrm{H}), 7.26(\mathrm{t}, J=6.7$ $\mathrm{Hz}, 2 \mathrm{H}), 2.45(\mathrm{~s}, 6 \mathrm{H}) ;{ }^{13} \mathrm{C} \mathrm{NMR}\left(\mathrm{CDCl}_{3}, 100 \mathrm{MHz}\right) \delta$ : $158.2,149.9,149.6,139.4,138.8,138.6,138.5,129.9$, $129.8,129.1,128.7,127.9,127.8,124.2,124.1,120.3$, $118.9,21.6,21.5$; IR (KBr) v: 1594, 1531, 1508, 1462, 1372, 784, $707 \mathrm{~cm}^{-1}$; HRMS (EI) calcd for $\mathrm{C}_{19} \mathrm{H}_{18} \mathrm{~N}$ 260.1434 , found 260.1435 .

2,4-Di-o-tolylpyridine (2f): Yellow oil, $27 \mathrm{mg}, 42 \%$ yield. ${ }^{1} \mathrm{H}$ NMR $\left(\mathrm{CDCl}_{3}, 400 \mathrm{MHz}\right) \delta: 8.77(\mathrm{~d}, J=5.0 \mathrm{~Hz}$, 1H), 7.45 (d, $J=7.0 \mathrm{~Hz}, 1 \mathrm{H}), 7.39$ (d, $J=4.5 \mathrm{~Hz}, 1 \mathrm{H}), 7.30$ $(\mathrm{t}, J=5.4 \mathrm{~Hz}, 5 \mathrm{H}), 7.26(\mathrm{~d}, J=9.2 \mathrm{~Hz}, 3 \mathrm{H}), 2.41(\mathrm{~d}, J=6.3$ $\mathrm{Hz}, 3 \mathrm{H}), 2.34(\mathrm{~s}, 3 \mathrm{H}) ;{ }^{13} \mathrm{C} \mathrm{NMR}\left(\mathrm{CDCl}_{3}, 100 \mathrm{MHz}\right) \delta$ : $159.6,150.3,148.8,140.1,139.1,135.8,135.1,130.8$, $130.7,129.8,129.4,128.5,128.4,126.2,126.0,124.8$, $122.4,20.5,20.4$; IR (KBr) v: 1594, 1541, 1456, 1386, $751,727 \mathrm{~cm}^{-1}$; HRMS (EI) calcd for $\mathrm{C}_{19} \mathrm{H}_{18} \mathrm{~N} 260.1434$, found 260.1438 .

2,6-Di-o-tolylpyridine (2f'): Yellow oil, $26 \mathrm{mg}, 40 \%$ yield. ${ }^{1} \mathrm{H}$ NMR $\left(\mathrm{CDCl}_{3}, 400 \mathrm{MHz}\right) \delta: 7.73(\mathrm{~d}, J=7.7 \mathrm{~Hz}$, $1 \mathrm{H}), 7.38$ (d, $J=8.9 \mathrm{~Hz}, 2 \mathrm{H}), 7.29$ (d, $J=7.7 \mathrm{~Hz}, 2 \mathrm{H})$, $7.22 \sim 7.16(\mathrm{~m}, 6 \mathrm{H}), 2.35(\mathrm{~s}, 6 \mathrm{H}) ;{ }^{13} \mathrm{C} \mathrm{NMR}\left(\mathrm{CDCl}_{3}, 100\right.$ $\mathrm{MHz}) \delta: 159.5,140.6,136.4,135.9,130.7,129.9,128.3$, 125.9, 122.1, 20.6; IR (KBr) v: 1601, 1562, 1457, 1374, $767 \mathrm{~cm}^{-1}$; HRMS (EI) calcd for $\mathrm{C}_{19} \mathrm{H}_{18} \mathrm{~N} 260.1434$, found 260.1435 .

2,4-Bis(3,4-dimethoxyphenyl)pyridine (2g): Yellow solid, $72 \mathrm{mg}, 82 \%$ yield. m.p. $111 \sim 113{ }^{\circ} \mathrm{C} ;{ }^{1} \mathrm{H}$ NMR $\left(\mathrm{CDCl}_{3}, 400 \mathrm{MHz}\right) \delta: 8.67(\mathrm{~d}, J=5.0 \mathrm{~Hz}, 1 \mathrm{H}), 7.84(\mathrm{~s}$, $1 \mathrm{H}), 7.71(\mathrm{~s}, 1 \mathrm{H}), 7.56(\mathrm{~d}, J=8.3 \mathrm{~Hz}, 1 \mathrm{H}), 7.38(\mathrm{~d}, J=5.0$ $\mathrm{Hz}, 1 \mathrm{H}), 7.28$ (d, $J=8.1 \mathrm{~Hz}, 1 \mathrm{H}), 7.19(\mathrm{~s}, 1 \mathrm{H}), 6.99$ (t, $J=$ $7.8 \mathrm{~Hz}, 2 \mathrm{H}), 4.02(\mathrm{~s}, 3 \mathrm{H}), 3.98(\mathrm{~s}, 3 \mathrm{H}), 3.95(\mathrm{~s}, 6 \mathrm{H}) ;{ }^{13} \mathrm{C}$ NMR $\left(\mathrm{CDCl}_{3}, 100 \mathrm{MHz}\right) \delta: 157.6,150.0,150.1,149.7$, $149.5,149.3,149.2,132.4,131.3,119.8,119.5,117.9$, $111.6,111.1,110.2,110.1,56.2,56.1,56.0,56.0$; IR (KBr) $v: 1597,1543,1466,1385,1237,1110,1057,982,943$, $815 \mathrm{~cm}^{-1}$; HRMS (EI) calcd for $\mathrm{C}_{21} \mathrm{H}_{22} \mathrm{NO}_{4} 352.1543$, found 352.1548 .

2,4-bis(2,4-dimethoxyphenyl)pyridine $\quad(\mathbf{2 h})$ : Yellow solid, $62 \mathrm{mg}, 71 \%$ yield. m.p. $115 \sim 118{ }^{\circ} \mathrm{C}$; ${ }^{1} \mathrm{H}$ NMR 
$\left(\mathrm{CDCl}_{3}, 400 \mathrm{MHz}\right) \delta: 8.65(\mathrm{~d}, J=5.1 \mathrm{~Hz}, 1 \mathrm{H}), 7.90(\mathrm{~s}$, $1 \mathrm{H}), 7.74(\mathrm{~d}, J=8.5 \mathrm{~Hz}, 1 \mathrm{H}), 7.35$ (dd, $J=14.4,6.7 \mathrm{~Hz}$, 2H), 6.60 (dd, $J=21.2,6.4 \mathrm{~Hz}, 4 \mathrm{H}), 3.85$ (s, 6H), 3.83 (s, $6 \mathrm{H}) ;{ }^{13} \mathrm{C} \mathrm{NMR}\left(\mathrm{CDCl}_{3}, 100 \mathrm{MHz}\right) \delta: 161.4,161.3,158.1$, $157.9,155.5,148.5,146.1,132.1,131.3,125.3,122.3$, 122.0, 121.0, 105.0, 99.1, 98.9, 55.7, 55.6, 55.5, 55.4; IR (KBr) v: 1592, 1536, 1451, 1391, 1232, 1121, 1050, 976, 938, $810 \mathrm{~cm}^{-1}$; HRMS (EI) calcd for $\mathrm{C}_{21} \mathrm{H}_{22} \mathrm{NO}_{4} 352.1543$, found 352.1547 .

2,4-Bis(4-fluorophenyl)pyridine (2i): White solid, 39 mg, 58\% yield, m.p. $78 \sim 81{ }^{\circ} \mathrm{C} ;{ }^{1} \mathrm{H}$ NMR $\left(\mathrm{CDCl}_{3}, 400\right.$ MHz) $\delta: 8.62(\mathrm{~d}, J=5.1 \mathrm{~Hz}, 1 \mathrm{H}), 7.95(\mathrm{dd}, J=8.0,5.7 \mathrm{~Hz}$, 2H), 7.74 (s, 1H), 7.57 (dd, $J=7.9,5.6 \mathrm{~Hz}, 2 \mathrm{H}), 7.31$ (d, $J=5.1 \mathrm{~Hz}, 1 \mathrm{H}), 7.10(\mathrm{q}, J=8.7 \mathrm{~Hz}, 4 \mathrm{H}) ;{ }^{13} \mathrm{C}$ NMR $\left(\mathrm{CDCl}_{3}, 100 \mathrm{MHz}\right) \delta: 164.8(\mathrm{~d}, J=13.5 \mathrm{~Hz}), 162.3(\mathrm{~d}, J=$ $14.2 \mathrm{~Hz}), 157.1,150.1,148.5,135.4$ (d, $J=3.0 \mathrm{~Hz}), 134.5$ $(\mathrm{d}, J=3.1 \mathrm{~Hz}), 128.9,128.8,120.0,118.3,116.2(\mathrm{~d}, J=$ $21.7 \mathrm{~Hz}), 115.7$ (d, $J=21.6 \mathrm{~Hz})$; IR (KBr) v: 1609, 1514, 1473, 1224, 1157, 816, $556 \mathrm{~cm}^{-1}$; HRMS (EI) calcd for $\mathrm{C}_{17} \mathrm{H}_{12} \mathrm{~F}_{2} \mathrm{~N} 268.0932$, found 268.0936.

2,4-Bis(4-chlorophenyl)pyridine (2j): White solid, 52 mg, 70\% yield. m.p. 101 104 ${ }^{\circ} \mathrm{C} ;{ }^{1} \mathrm{H}$ NMR $\left(\mathrm{CDCl}_{3}, 400\right.$ MHz) $\delta: 8.72(\mathrm{~d}, J=5.1 \mathrm{~Hz}, 1 \mathrm{H}), 7.98(\mathrm{~d}, J=8.1 \mathrm{~Hz}, 2 \mathrm{H})$, $7.84(\mathrm{~s}, 1 \mathrm{H}), 7.61(\mathrm{~d}, J=8.0 \mathrm{~Hz}, 2 \mathrm{H}), 7.47$ (t, $J=7.9 \mathrm{~Hz}$, 4H), $7.41(\mathrm{~d}, J=5.0 \mathrm{~Hz}, 1 \mathrm{H}) ;{ }^{13} \mathrm{C} \mathrm{NMR}\left(\mathrm{CDCl}_{3}, 100 \mathrm{MHz}\right)$ $\delta: 157.0,150.3,148.3,137.6,136.8,135.5,135.4,129.4$, 129.0, 128.4, 128.3, 120.3, 118.3; IR (KBr) v: 1598, 1542, $1495,1463,1417,1376,1090,1011,816,753 \mathrm{~cm}^{-1}$; HRMS (EI) calcd for $\mathrm{C}_{17} \mathrm{H}_{12} \mathrm{Cl}_{2} \mathrm{~N}$ 300.0341, found 300.0343 .

2,4-Bis(4-bromophenyl)pyridine (2k): White solid, 64 mg, 66\% yield. m.p. $137 \sim 139{ }^{\circ} \mathrm{C} ;{ }^{1} \mathrm{H}$ NMR $\left(\mathrm{CDCl}_{3}, 400\right.$ MHz) $\delta: 8.72(\mathrm{~d}, J=5.0 \mathrm{~Hz}, 1 \mathrm{H}), 7.92(\mathrm{~d}, J=8.3 \mathrm{~Hz}, 2 \mathrm{H})$, $7.84(\mathrm{~s}, 1 \mathrm{H}), 7.63(\mathrm{t}, J=8.4 \mathrm{~Hz}, 4 \mathrm{H}), 7.54(\mathrm{~d}, J=8.2 \mathrm{~Hz}$, 2H), $7.43 \sim 7.39(\mathrm{~m}, 1 \mathrm{H}) ;{ }^{13} \mathrm{C} \mathrm{NMR}\left(\mathrm{CDCl}_{3}, 100 \mathrm{MHz}\right) \delta$ : $157.0,150.3,148.4,138.1,137.2,132.4,132.0,128.7$, 128.6, 123.8, 123.7, 120.3, 118.2; IR (KBr) v: 1593, 1537, 1375, 1071, 1006, $812 \mathrm{~cm}^{-1}$; HRMS (EI) calcd for $\mathrm{C}_{17} \mathrm{H}_{12} \mathrm{Br}_{2} \mathrm{~N}$ 387.9331, found 387.9334.

2,4-Bis(3-bromophenyl)pyridine (2l): White solid, 53 mg, 54\% yield. m.p. $94 \sim 96{ }^{\circ} \mathrm{C}$; ${ }^{1} \mathrm{H}$ NMR $\left(\mathrm{CDCl}_{3}, 400\right.$ MHz) $\delta: 8.73(\mathrm{t}, J=7.9 \mathrm{~Hz}, 1 \mathrm{H}), 8.21(\mathrm{~s}, 1 \mathrm{H}), 7.97(\mathrm{~d}, J=$ $7.8 \mathrm{~Hz}, 1 \mathrm{H}), 7.82(\mathrm{~d}, J=9.1 \mathrm{~Hz}, 2 \mathrm{H}), 7.64 \sim 7.53(\mathrm{~m}, 3 \mathrm{H})$, $7.39(\mathrm{dt}, J=15.4,6.3 \mathrm{~Hz}, 3 \mathrm{H}) ;{ }^{13} \mathrm{C} \mathrm{NMR}\left(\mathrm{CDCl}_{3}, 100\right.$ $\mathrm{MHz}) \delta$ : $156.64,150.32,148.10,141.16,140.37,132.16$, $130.72,130.35,130.17,130.15,125.77,125.60,123.33$, $123.14,120.71,118.67$; IR (KBr) v: 1591, 1542, 1462, $783,706 \mathrm{~cm}^{-1}$; HRMS (EI) calcd for $\mathrm{C}_{17} \mathrm{H}_{12} \mathrm{Br}_{2} \mathrm{~N}$ 387.9331 , found 387.9335 .

2,4-Bis(2-bromophenyl)pyridine (2m): White solid, 33 mg, 34\% yield. m.p. $124 \sim 126{ }^{\circ} \mathrm{C} ;{ }^{1} \mathrm{H}$ NMR $\left(\mathrm{CDCl}_{3}, 400\right.$ MHz) $\delta: 8.81(\mathrm{~d}, J=5.1 \mathrm{~Hz}, 1 \mathrm{H}), 7.72 \sim 7.67(\mathrm{~m}, 3 \mathrm{H}), 7.62$ $(\mathrm{d}, J=7.8 \mathrm{~Hz}, 1 \mathrm{H}), 7.45 \sim 7.37(\mathrm{~m}, 4 \mathrm{H}), 7.29 \sim 7.23(\mathrm{~m}$, $2 \mathrm{H}) ;{ }^{13} \mathrm{C} \mathrm{NMR}\left(\mathrm{CDCl}_{3}, 100 \mathrm{MHz}\right) \delta: 158.0,148.9,140.7$, $139.8,133.5,133.4,132.3,131.6,130.9,130.0,129.9$, $127.8,127.6,125.6,123.2,121.9,121.8$; IR (KBr) v: 1601, $1564,1542,1458,1432,1387,1076,1023,899,755 \mathrm{~cm}^{-1}$;
HRMS (EI) calcd for $\mathrm{C}_{17} \mathrm{H}_{12} \mathrm{Br}_{2} \mathrm{~N}$ 387.9331, found 387.9336 .

2,6-Bis(2-bromophenyl)pyridine (2m'): Yellow oil, 37 mg, 38\% yield. ${ }^{1} \mathrm{H}$ NMR $\left(\mathrm{CDCl}_{3}, 400 \mathrm{MHz}\right) \delta: 7.84(\mathrm{t}, J=$ $7.8 \mathrm{~Hz}, 1 \mathrm{H}), 7.70 \sim 7.58(\mathrm{~m}, 6 \mathrm{H}), 7.40(\mathrm{t}, J=7.5 \mathrm{~Hz}, 2 \mathrm{H})$, $7.24(\mathrm{t}, J=7.7 \mathrm{~Hz}, 2 \mathrm{H}) ;{ }^{13} \mathrm{C} \mathrm{NMR}\left(\mathrm{CDCl}_{3}, 100 \mathrm{MHz}\right) \delta$ : $157.9,141.2,135.9,133.3,131.8,129.8,127.6,123.4$, 121.9; IR (KBr) v: 1637, 1384, 1184, 1126, 800, 688, 618 $\mathrm{cm}^{-1}$; HRMS (EI) calcd for $\mathrm{C}_{17} \mathrm{H}_{12} \mathrm{Br}_{2} \mathrm{~N}$ 387.9331, found 387.9333 .

2,4-Bis(4-iodophenyl)pyridine (2n): White solid, 65mg, $54 \%$ yield. m.p. $171 \sim 173{ }^{\circ} \mathrm{C}$; ${ }^{1} \mathrm{H}$ NMR $\left(\mathrm{CDCl}_{3}, 400\right.$ MHz) $\delta: 8.71(\mathrm{~d}, J=4.7 \mathrm{~Hz}, 1 \mathrm{H}), 7.91(\mathrm{~d}, J=8.3 \mathrm{~Hz}, 2 \mathrm{H})$, 7.83 (s, 1H), 7.62 (t, $J=8.1 \mathrm{~Hz}, 4 \mathrm{H}), 7.53(\mathrm{~d}, J=8.3 \mathrm{~Hz}$, $2 \mathrm{H}), 7.40(\mathrm{~d}, J=3.6 \mathrm{~Hz}, 1 \mathrm{H}) ;{ }^{13} \mathrm{C} \mathrm{NMR}\left(\mathrm{CDCl}_{3}, 100 \mathrm{MHz}\right)$ $\delta: 157.01,150.29,148.36,138.05,137.21,132.39,131.98$, $128.65,128.60,123.75,123.72,120.28,118.17$; IR (KBr) $v: 1596,1539,1372,1001,809 \mathrm{~cm}^{-1}$; HRMS (EI): calculated for $\mathrm{C}_{17} \mathrm{H}_{12} \mathrm{I}_{2} \mathrm{~N}$ 483.9054, found 483.9058 .

2,4-Bis(3-nitrophenyl)pyridine (2o): White solid, $28 \mathrm{mg}$, $35 \%$ yield. m.p. $194 \sim 197{ }^{\circ} \mathrm{C}$; ${ }^{1} \mathrm{H}$ NMR $\left(\mathrm{CDCl}_{3}, 400\right.$ MHz) $\delta: 8.94(\mathrm{~s}, 1 \mathrm{H}), 8.87(\mathrm{~d}, J=5.0 \mathrm{~Hz}, 1 \mathrm{H}), 8.57$ (s, $1 \mathrm{H}), 8.46$ (d, $J=7.8 \mathrm{~Hz}, 1 \mathrm{H}), 8.36(\mathrm{~d}, J=8.2 \mathrm{~Hz}, 1 \mathrm{H}), 8.32$ $(\mathrm{d}, J=8.1 \mathrm{~Hz}, 1 \mathrm{H}), 8.10 \sim 8.02(\mathrm{~m}, 2 \mathrm{H}), 7.80 \sim 7.68(\mathrm{~m}$, 2H), $7.59(\mathrm{~d}, J=4.8 \mathrm{~Hz}, 1 \mathrm{H}) ;{ }^{13} \mathrm{C} \mathrm{NMR}\left(\mathrm{CDCl}_{3}, 100 \mathrm{MHz}\right)$ $\delta: 155.9,150.8,148.9,148.9,147.5,140.5,139.8,133.1$, $132.9,130.4,129.9,124.1,124.0,122.1,122.0,121.3$, 118.7; IR (KBr) v: 1632, 1599, 1530, 1353, 815, 730, 677 $\mathrm{cm}^{-1}$; HRMS (EI) calcd for $\mathrm{C}_{17} \mathrm{H}_{12} \mathrm{~N}_{3} \mathrm{O}_{4} 322.0822$, found 322.0825

2,6-Bis(3-nitrophenyl)pyridine (2o'): White solid, $24 \mathrm{mg}$, $30 \%$ yield. m.p. $162 \sim 165{ }^{\circ} \mathrm{C}$; ${ }^{1} \mathrm{H}$ NMR $\left(\mathrm{CDCl}_{3}, 400\right.$ $\mathrm{MHz}) \delta: 8.96(\mathrm{~s}, 2 \mathrm{H}), 8.55(\mathrm{~d}, J=8.8 \mathrm{~Hz}, 2 \mathrm{H}), 8.32(\mathrm{~d}, J=$ $8.0 \mathrm{~Hz}, 2 \mathrm{H}), 7.99$ (d, $J=7.8 \mathrm{~Hz}, 1 \mathrm{H}), 7.89 \sim 7.83(\mathrm{~m}, 2 \mathrm{H})$, $7.72(\mathrm{t}, J=8.0 \mathrm{~Hz}, 2 \mathrm{H}) ;{ }^{13} \mathrm{C} \mathrm{NMR}\left(\mathrm{CDCl}_{3}, 100 \mathrm{MHz}\right) \delta$ : $154.8,148.9,140.6,138.5,132.9,130.0,124.0,121.8$, 120.0; IR (KBr) v: 1608, 1572, 1517, 1457, 1374, 1332, 786, $692 \mathrm{~cm}^{-1}$; HRMS (EI) calcd for $\mathrm{C}_{17} \mathrm{H}_{12} \mathrm{~N}_{3} \mathrm{O}_{4}$ 322.0822 , found 322.0824 .

4,4'-(Pyridine-2,4-diyl)dibenzonitrile (2p): White solid, $46 \mathrm{mg}, 66 \%$ yield, m.p. $175 \sim 178{ }^{\circ} \mathrm{C} ;{ }^{1} \mathrm{H} \mathrm{NMR}\left(\mathrm{CDCl}_{3}\right.$, $400 \mathrm{MHz}) \delta: 8.85(\mathrm{~d}, J=4.7 \mathrm{~Hz}, 1 \mathrm{H}), 8.19(\mathrm{~d}, J=8.1 \mathrm{~Hz}$, 2H), $7.94(\mathrm{~d}, J=6.2 \mathrm{~Hz}, 1 \mathrm{H}), 7.81(\mathrm{t}, J=7.1 \mathrm{~Hz}, 6 \mathrm{H}), 7.53$ $(\mathrm{d}, J=4.5 \mathrm{~Hz}, 1 \mathrm{H}) ;{ }^{13} \mathrm{C} \mathrm{NMR}\left(\mathrm{CDCl}_{3}, 100 \mathrm{MHz}\right) \delta$ : $156.26,150.80,147.86,142.98,142.49,133.04,132.69$, $132.63,127.91,127.65,127.53,121.36,119.11,113.16$, 112.92; IR (KBr) v: 2226, 1587, 1539, 1489, 1471, 1423, 1368, 1084, $818 \mathrm{~cm}^{-1}$; HRMS (EI) calcd for $\mathrm{C}_{17} \mathrm{H}_{12} \mathrm{~N}_{3}$ 282.1086 , found 282.1087 .

2,4-Bis(4-(trifluoromethyl)phenyl)pyridine (2q): White solid, $53 \mathrm{mg}, 58 \%$ yield, m.p. $69 \sim 70{ }^{\circ} \mathrm{C}$; ${ }^{1} \mathrm{H}$ NMR $\left(\mathrm{CDCl}_{3}, 400 \mathrm{MHz}\right) \delta: 8.81(\mathrm{~d}, J=5.0 \mathrm{~Hz}, 1 \mathrm{H}), 8.17(\mathrm{~d}, J=$ $8.1 \mathrm{~Hz}, 2 \mathrm{H}), 7.95(\mathrm{~s}, 1 \mathrm{H}), 7.80 \sim 7.75(\mathrm{~m}, 6 \mathrm{H}), 7.51(\mathrm{~d}, J=$ $5.0 \mathrm{~Hz}, 1 \mathrm{H}) ;{ }^{13} \mathrm{C} \mathrm{NMR}\left(\mathrm{CDCl}_{3}, 100 \mathrm{MHz}\right) \delta: 156.8,150.5$, $148.3,142.3,141.8,138.0,131.4$ (d, $J=15.3 \mathrm{~Hz}), 131.1$ $(\mathrm{d}, J=15.1 \mathrm{~Hz}), 127.6,127.4,126.2$ (q, $J=3.7 \mathrm{~Hz}), 125.8$ (q, $J=3.8 \mathrm{~Hz}), 121.1,119.8,119.1$; IR (KBr) $v$ : 1629, 
1551, 1492, 1471, 1425, 1382, 1324, 1176, $830 \mathrm{~cm}^{-1}$; HRMS (EI) calcd for $\mathrm{C}_{19} \mathrm{H}_{12} \mathrm{~F}_{6} \mathrm{~N}$ 368.0868, found 368.0869 .

2,4-Di(naphthalen-2-yl)pyridine (2r): Yellow solid, 56 mg, 68\% yield, m.p. $110 \sim 113{ }^{\circ} \mathrm{C} ;{ }^{1} \mathrm{H}$ NMR $\left(\mathrm{CDCl}_{3}, 400\right.$ $\mathrm{MHz}) \delta: 8.81(\mathrm{~d}, J=5.0 \mathrm{~Hz}, 1 \mathrm{H}), 8.57(\mathrm{~s}, 1 \mathrm{H}), 8.22(\mathrm{~d}, J=$ $8.6 \mathrm{~Hz}, 1 \mathrm{H}), 8.17(\mathrm{~s}, 2 \mathrm{H}), 7.95(\mathrm{t}, J=9.1 \mathrm{~Hz}, 4 \mathrm{H}), 7.90 \sim$ $7.84(\mathrm{~m}, 2 \mathrm{H}), 7.80(\mathrm{~d}, J=8.5 \mathrm{~Hz}, 1 \mathrm{H}), 7.57 \sim 7.48(\mathrm{~m}$, $5 \mathrm{H}) ;{ }^{13} \mathrm{C} \mathrm{NMR}\left(\mathrm{CDCl}_{3}, 100 \mathrm{MHz}\right) \delta: 157.9,150.1,149.5$, $136.6,135.7,133.8,133.6,133.5,129.0,128.8,128.6$, $128.5,127.8,127.7,126.9,126.8,126.7,126.6,126.6$, 126.4, 124.7, 124.7, 120.6, 119.3; IR (KBr) v: 1632, 1590, 1547, 828, 812, 756, $480 \mathrm{~cm}^{-1}$; HRMS (EI) calcd for $\mathrm{C}_{25} \mathrm{H}_{18} \mathrm{~N} 332.1434$, found 332.1436.

2,4-Di(thiophen-2-yl)pyridine (2s): Yellow solid, $32 \mathrm{mg}$, $52 \%$ yield. m.p. $55 \sim 58{ }^{\circ} \mathrm{C} ;{ }^{1} \mathrm{H} \mathrm{NMR}\left(\mathrm{CDCl}_{3}, 400 \mathrm{MHz}\right)$ $\delta: 8.54(\mathrm{~d}, J=5.2 \mathrm{~Hz}, 1 \mathrm{H}), 7.83(\mathrm{~s}, 1 \mathrm{H}), 7.68(\mathrm{~d}, J=3.2$ $\mathrm{Hz}, 1 \mathrm{H}), 7.55$ (t, $J=5.0 \mathrm{~Hz}, 1 \mathrm{H}), 7.44(\mathrm{dd}, J=12.1,6.2$ $\mathrm{Hz}, 2 \mathrm{H}), 7.35$ (d, $J=5.1 \mathrm{~Hz}, 1 \mathrm{H}), 7.19 \sim 7.12(\mathrm{~m}, 2 \mathrm{H}) ;{ }^{13} \mathrm{C}$ NMR $\left(\mathrm{CDCl}_{3}, 100 \mathrm{MHz}\right) \delta: 153.1,145.0,144.4,142.3$, $141.1,128.5,128.1,127.9,127.3,125.6,124.9,118.4$, 115.1; IR (KBr) v: 1594, 1539, 1470, 1422, 854, 826, 738, $709 \mathrm{~cm}^{-1}$; HRMS (EI) calcd for $\mathrm{C}_{13} \mathrm{H}_{10} \mathrm{~S}_{2} \mathrm{~N} 224.0249$, found 224.0252 .

3,5-Dimethyl-2,6-diphenylpyridine (3a): White solid, 42 mg, 65\% yield, m.p. 128 $131{ }^{\circ} \mathrm{C} ;{ }^{1} \mathrm{H}$ NMR $\left(\mathrm{CDCl}_{3}, 400\right.$ MHz) $\delta: 7.58(\mathrm{~d}, J=7.4 \mathrm{~Hz}, 4 \mathrm{H}), 7.47(\mathrm{~s}, 1 \mathrm{H}), 7.42(\mathrm{t}, J=$ $7.4 \mathrm{~Hz}, 4 \mathrm{H}), 7.35(\mathrm{t}, J=7.2 \mathrm{~Hz}, 2 \mathrm{H}), 2.37(\mathrm{~s}, 6 \mathrm{H}) ;{ }^{13} \mathrm{C}$ NMR $\left(\mathrm{CDCl}_{3}, 100 \mathrm{MHz}\right) \delta: 155.8,141.2,140.6,129.3$, 129.2, 128.1, 127.7, 19.6; IR (KBr) v: 1441, 1420, 1390, $775,763,702,607 \mathrm{~cm}^{-1}$; HRMS (EI) calcd for $\mathrm{C}_{19} \mathrm{H}_{18} \mathrm{~N}$ 260.1434 , found 260.1437 .

2,6-Bis(4-methoxyphenyl)-3,5-dimethylpyridine (3b): White solid, $57 \mathrm{mg}, 71 \%$ yield. m.p. $108 \sim 110{ }^{\circ} \mathrm{C} ;{ }^{1} \mathrm{H}$ NMR $\left(\mathrm{CDCl}_{3}, 400 \mathrm{MHz}\right) \delta: 7.46(\mathrm{~d}, J=8.0 \mathrm{~Hz}, 4 \mathrm{H}), 7.35$ (s, 1H), $6.86(\mathrm{~d}, J=8.1 \mathrm{~Hz}, 4 \mathrm{H}), 3.75(\mathrm{~s}, 6 \mathrm{H}), 2.29(\mathrm{~s}, 6 \mathrm{H})$; ${ }^{13} \mathrm{C} \mathrm{NMR}\left(\mathrm{CDCl}_{3}, 100 \mathrm{MHz}\right) \delta: 159.3,155.3,141.3,133.2$, $130.5,128.6,113.5,55.3,19.8$; IR (KBr) v: 1609, 1512, 1461, 1427, 1248, 1176, 1029, $837 \mathrm{~cm}^{-1}$; HRMS (EI) calcd for $\mathrm{C}_{21} \mathrm{H}_{22} \mathrm{NO}_{2} 320.1645$, found 320.1647.

3,5-Dimethyl-2,6-di-p-tolylpyridine (3c): Yellow oil, 49 mg, 68\% yield. m.p. $100 \sim 103{ }^{\circ} \mathrm{C} ;{ }^{1} \mathrm{H}$ NMR $\left(\mathrm{CDCl}_{3}, 400\right.$ MHz) $\delta: 7.40(\mathrm{~d}, J=7.7 \mathrm{~Hz}, 4 \mathrm{H}), 7.35(\mathrm{~s}, 1 \mathrm{H}), 7.14(\mathrm{~d}, J=$ $7.8 \mathrm{~Hz}, 4 \mathrm{H}), 2.30(\mathrm{~s}, 6 \mathrm{H}), 2.28(\mathrm{~s}, 6 \mathrm{H}) ;{ }^{13} \mathrm{C} \mathrm{NMR}\left(\mathrm{CDCl}_{3}\right.$, $100 \mathrm{MHz}) \delta$ : 155.7, 141.2, 137.3, 132.1, 129.9, 129.2, 128.7, 21.3, 19.7; IR (KBr) v: 1621, 1534, 1426, 908, 826, $732 \mathrm{~cm}^{-1}$; HRMS (EI) calcd for $\mathrm{C}_{21} \mathrm{H}_{22} \mathrm{~N} 288.1747$, found 288.1748 .

2,6-Bis(4-chlorophenyl)-3,5-dimethylpyridine (3d): White solid, $49 \mathrm{mg}, 60 \%$ yield. m.p. $138 \sim 139{ }^{\circ} \mathrm{C} ;{ }^{1} \mathrm{H}$ NMR $\left(\mathrm{CDCl}_{3}, 400 \mathrm{MHz}\right) \delta: 7.51(\mathrm{~d}, J=8.0 \mathrm{~Hz}, 4 \mathrm{H}), 7.49$ $(\mathrm{s}, 1 \mathrm{H}), 7.40(\mathrm{~d}, J=7.7 \mathrm{~Hz}, 4 \mathrm{H}), 2.36(\mathrm{~s}, 6 \mathrm{H}) ;{ }^{13} \mathrm{C} \mathrm{NMR}$ $\left(\mathrm{CDCl}_{3}, 100 \mathrm{MHz}\right) \delta: 154.6,141.6,138.7,133.9,130.6$, $129.5,128.4,19.6$; IR (KBr) v: 1608, 1557, 1495, 1454, 1093, 831, $767 \mathrm{~cm}^{-1}$; HRMS (EI) calcd for $\mathrm{C}_{19} \mathrm{H}_{16} \mathrm{NCl}_{2}$ 328.0654 , found 328.0656 .

2,6-Diphenyl-3,5-dipropylpyridine (3e): White solid, 42 mg, 54\% yield. m.p. $139 \sim 142{ }^{\circ} \mathrm{C} ;{ }^{1} \mathrm{H}$ NMR $\left(\mathrm{CDCl}_{3}, 400\right.$ MHz) $\delta: 7.52(\mathrm{~d}, J=7.5 \mathrm{~Hz}, 4 \mathrm{H}), 7.41(\mathrm{t}, J=7.3 \mathrm{~Hz}, 4 \mathrm{H})$, $7.38 \sim 7.32(\mathrm{~m}, 2 \mathrm{H}), 2.68 \sim 2.60(\mathrm{~m}, 4 \mathrm{H}), 1.65 \sim 1.53(\mathrm{~m}$, $4 \mathrm{H}), 0.90(\mathrm{t}, J=7.3 \mathrm{~Hz}, 6 \mathrm{H}) ;{ }^{13} \mathrm{C} \mathrm{NMR}\left(\mathrm{CDCl}_{3}, 100 \mathrm{MHz}\right)$ $\delta: 155.6,139.1,134.2,134.1,129.2,128.1,127.7,34.2$, 24.2, 14.0; IR (KBr) v: 2968, 1437, 1372, 775, 736, 699,

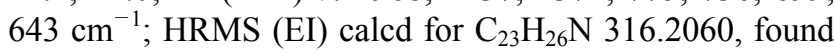
316.2058 .

(2,6-Diphenylpyridine-3,5-diyl)bis(phenylmethanone) (3f): White solid, $71 \mathrm{mg}, 65 \%$ yield, m.p. $181 \sim 184{ }^{\circ} \mathrm{C}$; ${ }^{1} \mathrm{H} \mathrm{NMR}\left(\mathrm{CDCl}_{3}, 400 \mathrm{MHz}\right) \delta: 8.05(\mathrm{~s}, 1 \mathrm{H}), 7.74(\mathrm{~d}, J=$ $7.8 \mathrm{~Hz}, 4 \mathrm{H}), 7.72 \sim 7.66(\mathrm{~m}, 4 \mathrm{H}), 7.46(\mathrm{t}, J=7.3 \mathrm{~Hz}, 2 \mathrm{H})$, $7.32(\mathrm{t}, J=7.8 \mathrm{~Hz}, 4 \mathrm{H}), 7.30 \sim 7.26(\mathrm{~m}, 6 \mathrm{H}) ;{ }^{13} \mathrm{C} \mathrm{NMR}$ $\left(\mathrm{CDCl}_{3}, 100 \mathrm{MHz}\right) \delta: 196.7,158.1,138.9,138.6,136.4$, $133.6,131.9,129.9,129.6,129.5,128.5,128.4$; IR (KBr) $v: 1663,1594,1528,1320,1247,1008,911,766,748,725$, $712,689 \mathrm{~cm}^{-1}$; HRMS (EI) calcd for $\mathrm{C}_{31} \mathrm{H}_{22} \mathrm{NO}_{2} 440.1645$, found 440.1647 .

5,6,8,9-Tetrahydrodibenzo[c,h]acridine $\quad(\mathbf{3 g})$ : Yellow solid, $45 \mathrm{mg}, 64 \%$ yield. m.p. $163 \sim 166{ }^{\circ} \mathrm{C} ;{ }^{1} \mathrm{H}$ NMR $\left(\mathrm{CDCl}_{3}, 400 \mathrm{MHz}\right) \delta: 8.51(\mathrm{~d}, J=7.6 \mathrm{~Hz}, 2 \mathrm{H}), 7.39(\mathrm{t}, J=$ $7.5 \mathrm{~Hz}, 2 \mathrm{H}), 7.30(\mathrm{t}, J=7.3 \mathrm{~Hz}, 3 \mathrm{H}), 7.23(\mathrm{t}, J=6.2 \mathrm{~Hz}$, 2H), $2.94(\mathrm{~s}, 8 \mathrm{H}) ;{ }^{13} \mathrm{C} \mathrm{NMR}\left(\mathrm{CDCl}_{3}, 100 \mathrm{MHz}\right) \delta: 150.4$, $137.9,135.3,135.0,130.6,128.6,127.7,127.1,125.0$, 28.3, 27.9; IR (KBr) v: 2922, 2832, 1552, 1435, 1418, 1237, 801, 747, $721 \mathrm{~cm}^{-1}$; HRMS (EI) calcd for $\mathrm{C}_{31} \mathrm{H}_{22} \mathrm{NO}_{2} 284.1434$, found 284.1435.

Supporting Information ${ }^{1} \mathrm{H}$ NMR and ${ }^{13} \mathrm{C}$ NMR spectra for all products. The Supporting Information is available free of charge via the Internet at http://sioc-journal.cn.

\section{References}

[1] (a) Eicher, T.; Hauptmann, S. The Chemistry of Heterocycles, Wiley-VCH, Weinheim, 2003.

(b) Katritzky, A. R.; Ramsden, C. A.; Scriven, E. F. V.; Taylor, R. J. Comprehensive Heterocyclic Chemistry, Elsevier, Oxford, 2008.

(c) Joule, J. A.; Mills, K. Heterocyclic Chemistry, Wiley, Chichester, 2010.

[2] (a) Forbes, I. T.; Johnson, C. N.; Jones, G. E.; Loudon, J.; Nicholass, J. M.; Thompson, M.; Upton, N. J. Med. Chem. 1990, 33, 2640.

(b) Zhmurenko, L. A.; Molodavkin, G. M.; Voronina, T. A.; Lezina, V. P. Pharm. Chem. J. 2012, 46, 15 .

[3] Bahekar, R. H.; Jain, M. R.; Jadav, P. A.; Prajapati, V. M.; Patel, D. N.; Gupta, A. A.; Sharma, A.; Tom, R.; Bandyopadhya, D.; Modi, H.; Patel, P. R. Bioorg. Med. Chem. 2007, 15, 6782.

[4] Rival, Y.; Grassy, G.; Taudou, A.; Ecalle, R. Eur. J. Med. Chem. 1991, 26, 13 .

[5] Zhuravel, I. O.; Kovalenko, S. M.; Ivachtchenko, A. V.; Balakin K. V.; Kazmirchuk, V. V. Bioorg. Med. Chem. Lett. 2005, 15, 5483.

[6] (a) Musonda, C. C.; Whitlock, G. A.; Witty, M. J.; Brun, R.; Kaiser, M. Bioorg. Med. Chem. Lett. 2009, 19, 401.

(b) Moreno, D.; Plano, D.; Baquedano, Y.; Jimeez-Ruiz, A.; Palop, J. A.; Sanmartn, C. Parasitol. Res. 2011, 108, 233.

[7] Rupert, K. C.; Henry, J. R.; Dodd, J. H.; Wadsworth, S. A.; Cavender, D. E.; Olini, G. C.; Fahmy, B.; Siekierka, J. J. Bioorg. Med. Chem. Lett. 2003, 13, 347.

[8] Kouznetsov, V. V.; Mendez, L. Y. V.; Tibaduiza, B.; Ochoa, C.; Pereira, D. M.; Ruiz, J. J. N.; Portillo, C. F.; Serrano, S. M.; Barrio, A. G.; Bahsas, A.; Amaro-Luis, J. Arch. Pharm. Pharm. Med. Chem. 
$\mathbf{2 0 0 4}, 337,127$.

[9] (a) Gibson, V. C.; Redshaw, C.; Solan, G. A. Chem. Rev. 2007, 107, 1745.

(b) Rycke, N. D.; Couty, F.; David, O. R. P. Chem.-Eur. J. 2011, 17, 12852.

[10] (a) Allais, C.; Grassot, J. M.; Rodriguez, J.; Constantieux, T. Chem. Rev. 2014, 114, 10829.

(b) Zhao, M. N.; Hui, R. R.; Ren, Z. H.; Wang, Y. Y.; Guan, Z. H. Org. Lett. 2014, 16, 3082.

(c) Bai, Y.; Tang, L. C.; Huang, H. W.; Deng, G. J. Org. Biomol. Chem. 2015, 13, 4404.

(d) Yan, Y. Z.; Li, H. Y.; Li, Z.; Niu, B.; Shi, M. M.; Liu, Y. Q. J. Org. Chem. 2017, 82, 8628.

[11] (a) Meunier, B. Biomimetic Oxidations Mediated by Metal Complexes, Imperial College Press, London, 2000.

(b) Meunier, B.; de Visser, S. P.; Shaik, S. Chem. Rev. 2004, 104, 3947.

(c) Zhou, X. T.; Ji, H. B.; Pei, L. X.; She, Y. B.; Xu, J. C.; Wang, L. F. Chin. J. Org. Chem. 2007, 27, 1039 (in Chinese).

(周贤太, 纪红兵, 裴丽霞, 余远斌, 徐建昌, 王乐夫, 有机化学, 2007, 27, 1039.)

[12] (a) Collman, J. P.; Wang, Z.; Straumanis, A.; Quelquejeu, M.; Rose, E. J. Am. Chem. Soc. 1999, 121, 460. (b) Yu, X. Q.; Huang, J. S.; Yu, W. Y.; Che, C. M. J. Am. Chem. Soc. 2000, 122, 5337.

(c) Au, S. M.; Huang, J. S.; Yu, W. Y.; Fung, W. H.; Che, C. M. J. Am. Chem. Soc. 1999, 121, 9120.

[13] (a) Li, Y.; Huang, J. S.; Zhou, Z. Y.; Che, C. M. J. Am. Chem. Soc. 2001, 123, 4843 .

(b) Bartoli, J. F.; Mansuy, V. M.; Barch-Ozette, K. L.; Palacio, M.; Battioni, P.; Mansuy, D. Chem. Commun. 2000, 827.

(c) Yang, J.; Weinberg, R.; Breslow, R. Chem. Commun. 2000, 531.

(d) Yu, W. Y.; Huang, J. S.; Zhou, Z. Y.; Che, C. M. Org. Lett. 2000, 2,2233

[14] (a) Li, Y. F.; Guo, C, C.; Yan, X. H.; Liu, Q. J. Porphyrins Phthalocyanines 2006, 10, 942 .

(b) Jiang, Q.; Sheng, W. B.; Tian, M.; Tang, J. S.; Guo, C. C. Eur. J. Org. Chem. 2013, 10, 1861.

(c) Sheng, W. B.; Jiang, Q.; Luo, W. P.; Guo, C. C. J. Org. Chem. 2013, 78, 5691.

[15] (a) Pan, X. J.; Liu, Q.; Chang, L. M.; Yuan, G. Q. RSC $A d v$. 2015, 5, 51183.

(b) Wu, X.; Zhang, J. H.; Liu, S.; Gao, Q. H.; Wu, A. X. Adv. Synth. Catal. 2016, 358, 218.

[16] Liu, Y. F.; Ji, P. Y.; Xu, J. W.; Hu, Y. Q.; Liu, Q.; Luo, W. P.; Guo, C. C. J. Org. Chem. 2017, 82, 7159.

(Zhao, C.) 